\title{
Modification of Membrane Fluidity by Gravity
}

\author{
Michaela Sieber, Wolfgang Hanke, Florian P. M. Kohn \\ Institute of Physiology, University of Hohenheim, Stuttgart, Germany \\ Email: wolfgang.hanke@uni-hohenheim.de
}

Received 11 July 2014; revised 11 August 2014; accepted 10 September 2014

Copyright (C) 2014 by authors and Scientific Research Publishing Inc.

This work is licensed under the Creative Commons Attribution International License (CC BY). http://creativecommons.org/licenses/by/4.0/

c) (i) Open Access

\begin{abstract}
Biological membranes are preferentially composed of lipids and proteins, and it is assumed that mainly the proteins are responsible for their functional properties. Nevertheless, during the last years, the contribution of the plain lipid matrix and its physico-chemical parameters to membrane functionality has been shown to be of high relevance. This is also correct for the gravity dependence of cells and organisms which is well accepted since long for a wide range of biological systems. Thus, the question must be asked, whether, and how far plain lipid membranes are affected by gravity directly. In this study we show that the fluidity (viscosity) of plain lipid membranes, as well as that of cell membranes, is gravity dependent, using a multipurpose 96-well plate reader in the fluorescence polarization anisotropy mode in a parabolic flight mission. Plain lipid vesicles and cells from a human cancer cell line have been used in these experiments. Necessarily, membrane-integrated proteins should be affected by this in their function. As a consequence any living cell will be able to sense at least basically gravity.
\end{abstract}

\section{Keywords}

Microgravity, Cells, Liposomes, Membrane Fluidity, Fluorescence Polarisation Anisotropy

\section{Introduction}

Biological membranes are preferentially composed of lipids and proteins, and it is assumed that mainly the proteins are responsible for their functional properties, and thus for a possible gravity dependence.

Gravity indeed is known to influence a lot of physical and biological processes. Biological cells and their reactions are no exception. Among others, the behavior of nerve cells and their gravity dependence is very important with the perspective of manned space missions. Previous experiments with vertebrates showed that, for example the speed of neuronal impulses in nerve fibers is slowing down under zero gravity and the nerve pulses 
are running faster under higher gravitation [1]. There are many ideas about the changed properties of nervous systems under microgravity, but the molecular principles are largely unexplored. In previous studies additional results have been obtained for different ion channels, which show a changed activity under microgravity [2]-[4]. Such changes in ion-channel activity might be well responsible for changes in higher cellular function. Up to now, however, it is still open whether these changes are due to direct interaction of gravity with the channel forming proteins, or whether gravity interacts with the lipid core of the membrane and thus changes the physical state of the membrane. A change, for example, in membrane fluidity could result in the change of function of ion-channels. The same would be true for the function of all other integral membrane proteins under conditions of variable gravity.

No direct information is given up to now concerning this question, however, some first results from droptower experiments have been published, showing that the size of plain lipid vesicles and thus their membrane area is gravity dependent, increasing towards smaller gravity values [5]. According to thermodynamical principles, the fluidity of the lipid membranes of these liposomes should increase under microgravity.

A well-know approach to study membrane fluidity directly is the use of fluorescent dyes together with the fluorescence polarization anisotropy (FPA) technique [6] [7]. Consequently, we have used a multipurpose 96well plate reader in the FPA mode with DPH (1,6-Diphenyl-1,3-5-Hexatriene) as a fluorescent dye in a parabolic flight mission to investigate the above stated question. Plain lipid vesicles, as well as human neuronal cells for comparison, have been used in this study.

\section{Materials and Methods}

\subsection{Materials}

All substances and solvents used in the experiments and not stated separately above were purchased from Sigma-Aldrich at least of p.a. grade. Distilled water was from a laboratory distillery. Cell culture medium was purchased from Biochrom.

\subsection{Vesicle Preparation}

Asolectin, a lipid extract from soybean membranes enriched in phosphatidylcholine, was purchased from Sigma-Aldrich. Di-myristoyl-phosphatidylcholine (DMPC) was purchased from Avanti Polar Lipids. This lipid has a main phase transition at about $24^{\circ} \mathrm{C}$.

For the preparation of the vesicles, lipids $(20 \mathrm{mg} / \mathrm{ml})$ were dissolved in a solvent-mixture (chloroform: methanol 2:1). The fluorescent dye DPH $(10 \mu \mathrm{M}$, dissolved in Chloroform) was also added. $9 \mathrm{ml}$ of this solution were filled in a flask and the solvent-mixture was removed in a rotary evaporator ( $400 \mathrm{mbar}, 1 \mathrm{~h}$ ) until the lipid was a thin film. To remove the last residue of solvent, the flask was stored in an exsiccator for $2 \mathrm{~h}$. Afterwards the lipid-film was rehydrated with $9 \mathrm{ml}$ of buffer $(100 \mathrm{mM} \mathrm{KCl}, 10 \mathrm{mM}$ Tris, $\mathrm{pH}$ 7,4), until the lipid has completely come off the wall. The solution was stored overnight in a fridge. The rehydrated lipid was sonicated for $30 \mathrm{~min}$ and afterwards centrifuged for $10 \mathrm{~min}$ (19000 rpm). For a usable vesicle concentration, the supernatant was diluted 1:10 with buffer. All the procedures were done with a minimum of light.

The liposomes were prepared before the flight and $300 \mu \mathrm{l}$ of liposome solution were filled into the wells of the plate. Additionally the reference wells were filled with aqueous solution, and then the plate was tightly sealed by an adhesive plastic foil. For the liposome measurements the plate reader was set to the top-read mode.

\subsection{Cell Culture}

The human neuroblastoma cell line SH-SY5Y was used for this experiment [8] [9], in its undifferentiated state and after 6-day differentiation with retinoic acid to neuron-like cells [10]. The cells were cultured in DMEMHam's F12 medium complemented with 10\% FCS, 100 Units/ml penicillin, $100 \mu \mathrm{g} / \mathrm{ml}$ streptomycin in T25 flasks at $37^{\circ} \mathrm{C}$ and $5.5 \% \mathrm{CO}_{2} .24$ hours before the flight the cells were transferred to 96-well plates with clear bottom. For an optimal fluorescence yield it was aimed at confluent mono-layers in the 96 wells. 2 hours before take-off, during the dyeing protocol, the culture medium was replaced with Krebs-Ringer-Hepes-buffer (KRH) of the following composition: $5 \mathrm{mM} \mathrm{KCl}, 130 \mathrm{mM} \mathrm{NaCl}, 2 \mathrm{mM} \mathrm{CaCl} 2,1.2 \mathrm{mM} \mathrm{MgSO}_{4}\left(7 \mathrm{H}_{2} \mathrm{O}\right), 1.2 \mathrm{mM}$ $\mathrm{KH}_{2} \mathrm{PO}_{4}, 6 \mathrm{mM}$ D-Glucose-monohydrate, $25 \mathrm{mM}$ Hepes. The solution was set to $\mathrm{pH} 7.4$ with $\mathrm{NaOH}$, the osmolarity was determined to be $\sim 300$ mOsm. 
The cells were visualized and inspected for viability and adherence by a microscope before and after the flights. Detachment of cells was never observed.

\subsection{Assay Preparation for DPH}

DPH was dissolved in DMSO to a stock-solution of $8 \mathrm{mM}$. The stock-solution was frozen in $5 \mu \mathrm{l}$ aliquots. For loading the cells, the stock-solution was mixed with KRH-buffer to obtain a final concentration of $8 \mu \mathrm{M}$ in each well. For a 96-well plate $5 \mu$ stock-solution was mixed with $4995 \mu \mathrm{l} \mathrm{KRH-buffer.}$

The dyeing protocol was as follows: The cell culture medium was removed from the wells and replaced with $200 \mu \mathrm{l} \mathrm{KRH-buffer,} \mathrm{and} \mathrm{then} \mathrm{it} \mathrm{was} \mathrm{washed} \mathrm{twice} \mathrm{with} 200 \mu \mathrm{l}$ KRH-buffer. Following that $50 \mu \mathrm{l}$ of the DPHKRH-solution were added per well and the plate was incubated in darkness for 45 minutes at room temperature. Then each well was washed twice with $200 \mu \mathrm{KRH}$-buffer, avoiding strong light and finally filled completely with KRH-buffer. After this procedure the plates were stored in darkness.

\subsection{Sealing Procedures}

The wells were completely filled with vesicle buffer or KRH respectively to avoid air bubbles which could disturb the recording due to their movement under microgravity. To avoid liquid leakage during microgravity the whole 96-well plate had to be sealed with sealing foil, suitable for fluorescence applications.

\subsection{FPA Measurements}

All experiments were carried out in a multipurpose 96-well plate reader from BMG-LabTech (PHERAstar FS, http://www.bmglabtech.com/en/products/microplate-readers/). The reader was properly housed for the parabolic flights, following the safety-rules given in the related documents [11] [12].

The reader was used in the FPA mode with the fluorescent dye DPH [13]. DPH was purchased from Sigma-Aldrich. All FPA cell experiments were conducted in the bottom read mode in the kinetic mode. The timing was set to 25 samples per well and parabola, for more details see the results section.

One row of a 96-well plate was used for each parabola with 9 wells filled with liposomes or cells, and three wells used as reference with plain aqueous solution. In Figure 1 the situation is demonstrated in some detail.

Due to the auto-gain function of the plate reader, the wells which were used as references were measured at a much higher gain. In these wells no liposomes were present, but only pure Ringer solution with and without $\mathrm{DPH}$. In both cases it is known that the fluorescence is very low, this was controlled in the lab, thus the traces in flight are taken at very high gain and the absolute values cannot be compared to the data traces directly.

Each recording started with about 20 seconds in $1 \mathrm{~g}$ and lasted until $1 \mathrm{~g}$ was reached again after the parabola. According to the timeline of DLR parabolic flights [11] [12] a single parabola can be divided into two phases of approximately 20 seconds of macrogravity (about $1.8 \mathrm{~g}$ ) and one phase of about 22 seconds of microgravity.

\subsection{Data Evaluation}

The data were stored on computer by the reader specific software from BMG LabTech, and later offline evaluated in a first step using the Mars ${ }^{\circledR}$ software from BMG. The 9 data traces for each parabola were averaged as well as the three reference traces. In case necessary, distorted data and outliers were excluded. Then, data traces were normalized with the reference traces, as given by the Mars ${ }^{\circledR}$ software. Finally all normalized traces for each condition were averaged again. This and the final data presentation were done using the Origin ${ }^{\circledR}$ software. The numbers of the different experiments performed are given in the results section. From the final traces, relative numbers (compared to $1 \mathrm{~g}$ ) of fluorescence polarization for each gravity condition were taken and plotted as function of gravity value.

\section{Results}

A basic set of processed data from an experiment is given in Figure 2. The upper graph shows averaged original data traces as obtained during an Asolectin vesicle experiment. The upper trace in this graph is from the reference wells, the lower one presents the Asolectin data points. The graph below shows the normalized data. The duration of the microgravity and macro gravity phases are in the range of 20 seconds, the $1 \mathrm{~g}$ phases last from 1 

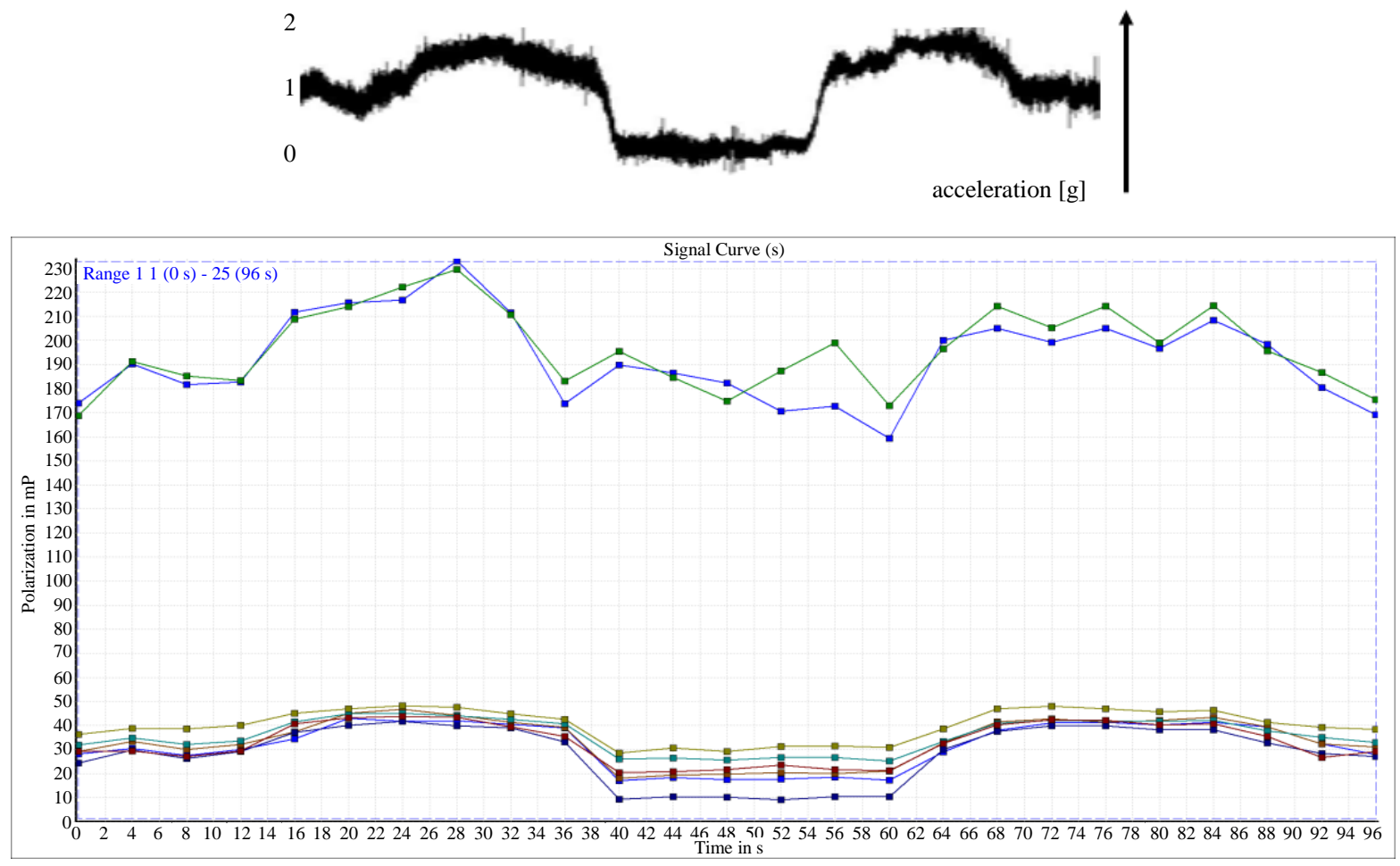

Figure 1. Original recording from a 96-well plate (lower part). The upper traces are two control wells; the lower traces are 6 vesicle traces from one row recorded during one parabola. Data of distorted wells and outliers from the row were excluded. The gravity profile of the parabola can be clearly seen in the traces, in comparison with the gravity profile of the parabola as given above the plate data. The reference traces are measured at a higher gain (auto-gain of the reader) which can be seen in the significant bigger scatter.
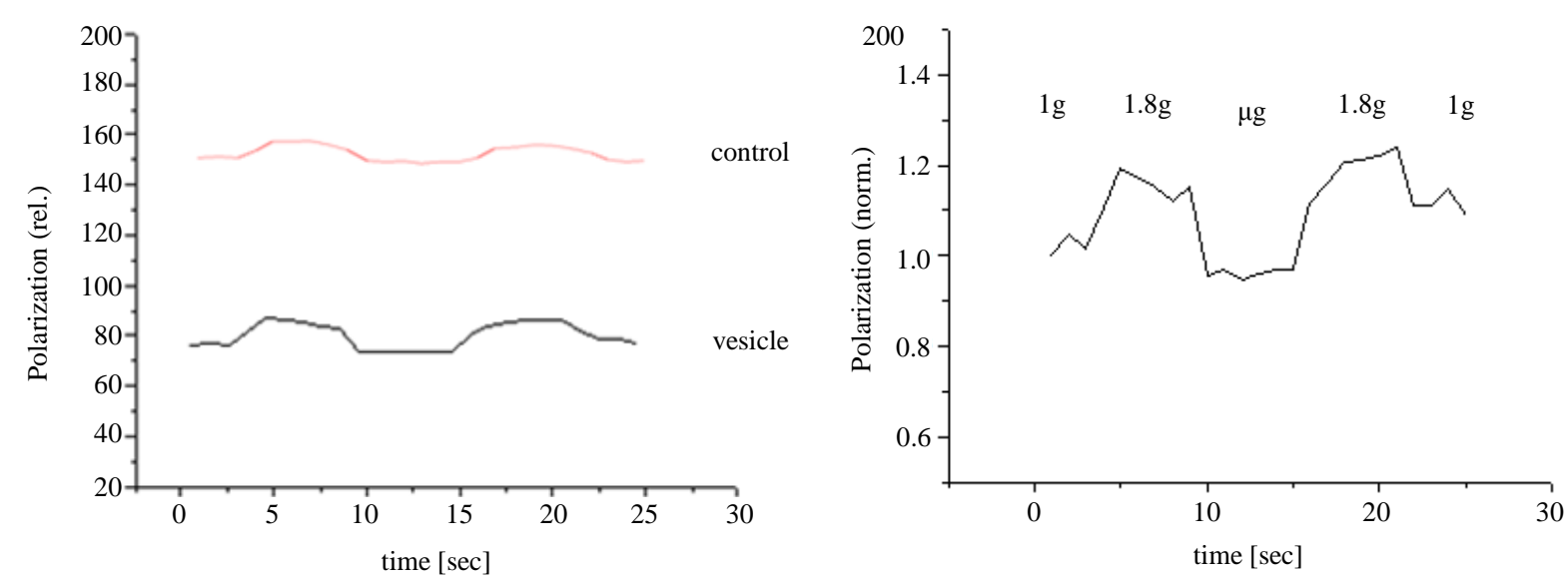

Figure 2. Upper part, set of processed (filtered and averaged) original data, the upper trace shows the control wells, the lower one the wells with Asolectin vesicles. Clearly the gravity response of the system can be seen. In the lower part the Asolectin data are shown again, normalized by the reference trace. It is obvious that the polarization is lower at lower acceleration.

to about 10 min between the separate parabolas. 31 parabolas are done during one flight. Details are given in the material section and the literature [11] [12].

\subsection{Experiments with Liposomes}

In a first set of experiments we investigated the influence of gravity on the membrane fluidity of Asolectin vesicles at a temperature around $23^{\circ} \mathrm{C}$ using fluorescence polarization (FP) of DPH. At this temperature these 
membranes are definitely in the fluid state. The data from 2 flight days of a parabolic flight campaign are presented in Figure 3. Obviously fluorescence polarization increases towards higher gravity. Thus, towards this direction membrane fluidity is decreasing, membranes are more fluid (lower viscosity) at lower gravity.

Next, we repeated the FP experiments with vesicles made from DMPC at $24^{\circ} \mathrm{C}$, thus the membranes of these vesicles are in the range of the phase transition. The data are presented in Figure 4. It is obvious that FP is not significantly dependent on gravity in this case. According to these experiments one should have in mind that membrane compressibility is highest in the range of phase transitions.

We also tried to perform experiments with frozen membranes, DMPC at temperatures significantly lower than $23^{\circ} \mathrm{C}$, but were not able to get reasonable results here, due to the fact that stable cooling of the PheraStar was not possible on board of the A300.

\subsection{Experiments with SH-SY5Y Cells}

Finally we performed a series of experiments with SH-SY5Y cells in the normal and in the re-differentiated state at about $23^{\circ} \mathrm{C}$. In both cases FP increased towards higher gravity, but less than with Asolectin vesicles. Furthermore the gravity dependence membrane fluidity of both cell types was different. The data are presented in Figure 5.

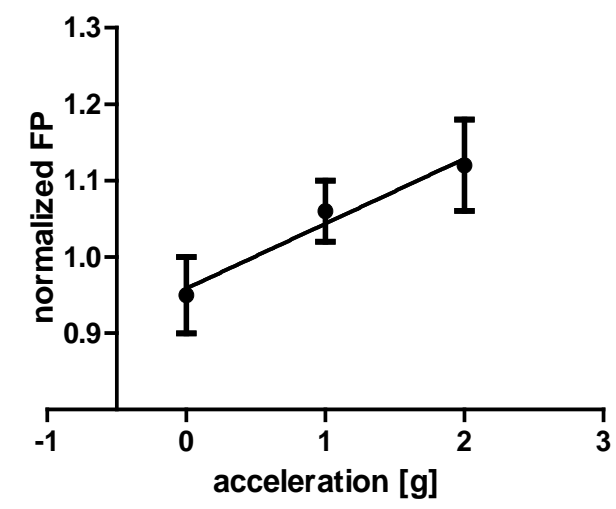

Figure 3. Gravity dependence of the fluorescence polarization (FP) of DPH doped Asolectin vesicles at about $23^{\circ} \mathrm{C}$, thus the vesicle membranes are in the fluid state. FP increases with increasing acceleration, accordingly membranes get more fluid at lower acceleration. Each data point at least presents the data of at least 20 parabola. Mean \pm SD are shown in the graph.

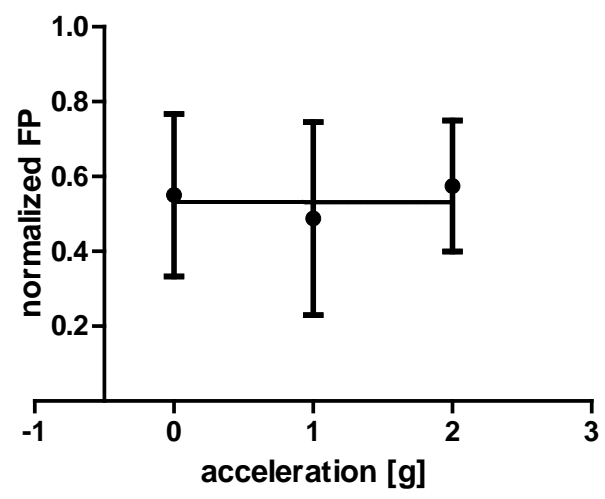

Figure 4. Gravity dependence of the fluorescence polarization (FP) of DPH doped DMPC vesicles at about $24^{\circ} \mathrm{C}$, thus the vesicle membranes are in the phase transition range. FP is not significantly affected by gravity. Each data point at least presents the data of 10 parabola. Mean \pm SD are shown in the graph. 


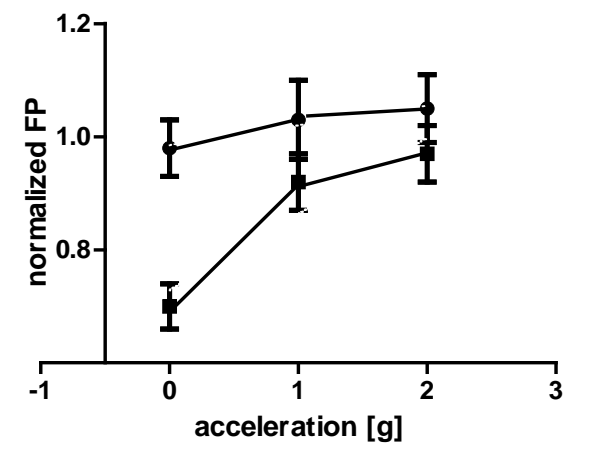

- differentiated cells

- not differentiated cells

Figure 5. Gravity dependence of the fluorescence polarization (FP) of DPH doped SH-SY5Y cells, differentiated and not differentiated (see methods section). In both cases, FP increases with increasing gravity, less than with Asolectin vesicles and different between the two cell types. The latter indicating a changed membrane composition as a consequence of re-differentiation. Each data point represents the results of at least 10 parabola. Mean \pm SD are shown in the graph.

From the data it is obvious that membrane the fluidity of SH-SY5Y cells significantly increases at microgravity.

\section{Discussion}

As it is obvious from the results, plain lipid membranes of vesicles in the fluid state are becoming more fluid at lower gravity values (the viscosity decreases towards this direction). Although the absolute values are difficult to interpret, the trend is obvious and significant. This finding is consistent with earlier results [5] showing a slight increase of vesicle size (increase in membrane area) of plain lipid vesicles under microgravity. Experiments with liposomes in the range of the phase transition temperature of the used lipid (DMPC, $24^{\circ} \mathrm{C}$ ) gave no significant gravity dependence of membrane fluidity. According to the fact that the compressibility of lipid membranes is highest in the phase transition range [14], this finding is consistent with thermodynamic rules. Experiments with liposomes in the crystalline state were not possible under the given experimental conditions.

Amazingly also the fluidity of cell membranes (SH-SY5Y, both types) significantly changes under different gravity conditions, again the fluidity increases at lower gravity values. This should be reflected again by an increase in membrane area of the cells, and thus in cell size, which however, up to now has not been reported. The existence of a cell cytoskeleton should, at least during fast changes, inhibit a significant change in cell size, too. Thus, a possible increase in membrane area might or must lead to a higher structured surface of the cells. Such an effect should be investigated in future experiments. The difference in the behavior of standard SH-SY5Y cell and re-differentiated cells cannot fully be explained at present. Nevertheless, both cell types at least changed towards the same direction. The difference then in principle might be a consequence of changes in membrane composition during re-differentiation [10].

The consequences of our results are manifold. The changes in ion-channel properties under changing gravity [1] [4] could at least be partially the result of the change of membrane properties, as it is well established that a variety of ion-channels are depending in their properties on the physical state of the membrane [15].

Our findings furthermore point out a more general dependence of membrane function on gravity, as about all membrane proteins will depend in their function on membrane thermodynamics, too. Finally, higher membrane functions, and their dependence on gravity, i.e. the propagation of action potentials [16] [17], might be influenced this way. Basically, as a consequence of our results, any biological cell must be considered to have at least some residual gravity sensitivity.

A variety of pharmacological interactions are based on membrane related processes, also in the field of spacepharmacology [18]. Our finding point out some new important questions which should be considered in future experiments, among them being receptor-ligand interaction, and especially the incorporation of hydrophobic and amphiphilic substances into membranes. Changes in incorporation of substances into membranes might have a significant influence on the pharmacological action of such substances. 


\section{Acknowledgements}

This work was supported by Deutsches Zentrumfür Luft-und Raumfahrt (DLR) grant 50WB1022.

\section{References}

[1] Wiedemann, M., Kohn, F.P.M., Rösner, H. and Hanke, W. (2011) Self-Organization and Pattern-Formation in Neuronal Systems under Conditions of Variable Gravity. In: Higher Education Press, Springer Publishing Company, Beijing.

[2] Goldermann M. and Hanke W. (2001) Ion-Channels Are Sensitive to Gravity Changes. Microgravity Science and Technology, 13, 35-38. http://dx.doi.org/10.1007/BF02873330

[3] Klinke, N., Goldermann, M. and Hanke, W. (2000) The Properties of Alamethicin Incorporated into Planar Lipid Bilayers under the Influence of Microgravity. Acta Astronautica, 47, 771-773. http://dx.doi.org/10.1016/S0094-5765(00)00115-6

[4] Wiedemann, M., Rahmann, H. and Hanke, W. (2003) Gravitational Impact on Ion Channels Incorporated into Planar Lipid Bilayers. In: TiTien and Ottova, Eds., Planar Lipid Bilayers and Their Applications, Elesevier Sciences, Amsterdam, 669-698. http://dx.doi.org/10.1016/S0927-5193(03)80048-7

[5] Meissner, K., Piqueira, J.R.C. and Hanke, W. (2004) Flourescent and Dispersion Experiments on Biological Membranes under Micro-Gravity. Journal of Gravitational Physiology, 11, 195-196.

[6] Lakowicz, J.R. (2006) Principles in Fluorescence of Macromolecules. Springer, New York, Berlin. http://dx.doi.org/10.1007/978-0-387-46312-4

[7] Fuchs, P., Parola, A., Robbins, P.W. and Blout, E.R. (1975) Fluorescence Polarization and Viscosity of Membrane Lipids of 3T3 Cells. Proceedings of the National Academy of Sciences, 72, 3351-3354. http://dx.doi.org/10.1073/pnas.72.9.3351

[8] Tosetti, P., Taglietti, V. and Toselli, M. (1998) Functional Changes in Potassium Conductances of the Human Neuroblastoma Cell Line SH-SY5Y during in Vitro Differentiation. Journal of Neurophysiology, 79, 648-658

[9] Biedler, J.L., Helson, L. and Spengler, B.A. (1973) Morphology and Growth, Tumorigenicity, and Cytogenetics of Human Neuroblastoma Cells in Continuous Culture. Cancer Research, 33, 2643-2652.

[10] Abemayor, E. (1992) The Effects of Retinoic Acid on the in Vitro and in Vivo Growth of Neuroblastoma Cells. Laryngoscope, 102, 1133-1149. http://dx.doi.org/10.1288/00005537-199210000-00008

[11] Novespace (2007) Parabolic Flight Campaign: Practical and Technical Information. DI-2007-3-en.

[12] Novespace (2009) A300 Zero-G Rules and Guidelines. RG-2009-2.

[13] Haugland, R.P. (2005) The Handbook: A Guide to Fluorescent Probes and Labeling Technologies. Molecular Probes (Invitrogen), Carlsbad, CA, USA.

[14] Blicher, A., Wodzinska, K., Fidorra, M., Winterhalter, M. and Heimburg, T. (2009) The Temperature Dependence of Lipid Membrane Permeability, Its Quantized Nature and the Influence of Anesthetics. Biophysical Journal, 96, 45814591. http://dx.doi.org/10.1016/j.bpj.2009.01.062

[15] Sachs, F. (2010) Stretch-Activated Ion Channels: What Are They? Physiology, 25, 50-56. http://dx.doi.org/10.1152/physiol.00042.2009

[16] Meissner, K. and Hanke, W. (2005) Action Potential Properties Are Gravity Dependent. Microgravity Science \& Technology, 17, 38-43. http://dx.doi.org/10.1007/BF02870977

[17] Rüegg, D.G., Kakebeeke, T.H. and Studer, L.M. (2000) Einfluss der Schwerkraft auf die Fortleitungsgeschwindigkeit von Muskel-Aktionspotentialen. In: Kelle, H. and Sahm, P.R., Eds., Bilanzsymposium Forschung unter Weltraumbedingungen, WPF, Aachen, 752-759.

[18] Wotring, V.E. (2012) Space Pharmacology (Springer Briefs in Space Development). Springer, New York. 
Scientific Research Publishing (SCIRP) is one of the largest Open Access journal publishers. It is currently publishing more than 200 open access, online, peer-reviewed journals covering a wide range of academic disciplines. SCIRP serves the worldwide academic communities and contributes to the progress and application of science with its publication.

Other selected journals from SCIRP are listed as below. Submit your manuscript to us via either submit@scirp.org or Online Submission Portal.
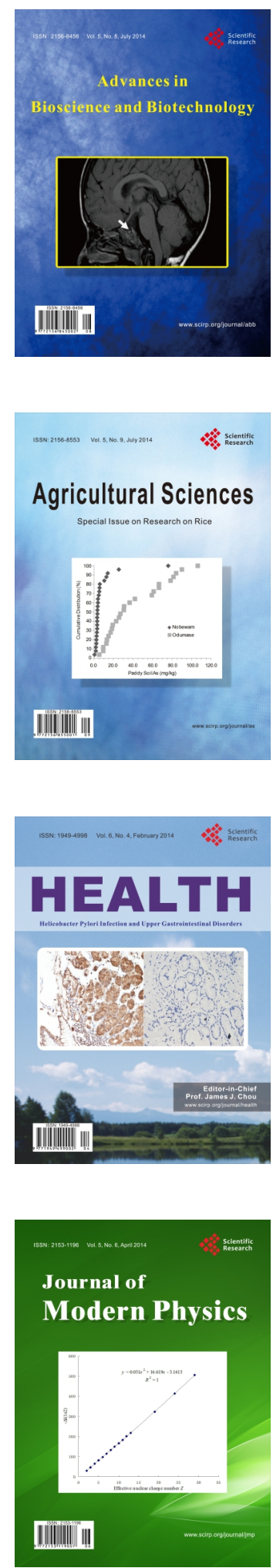
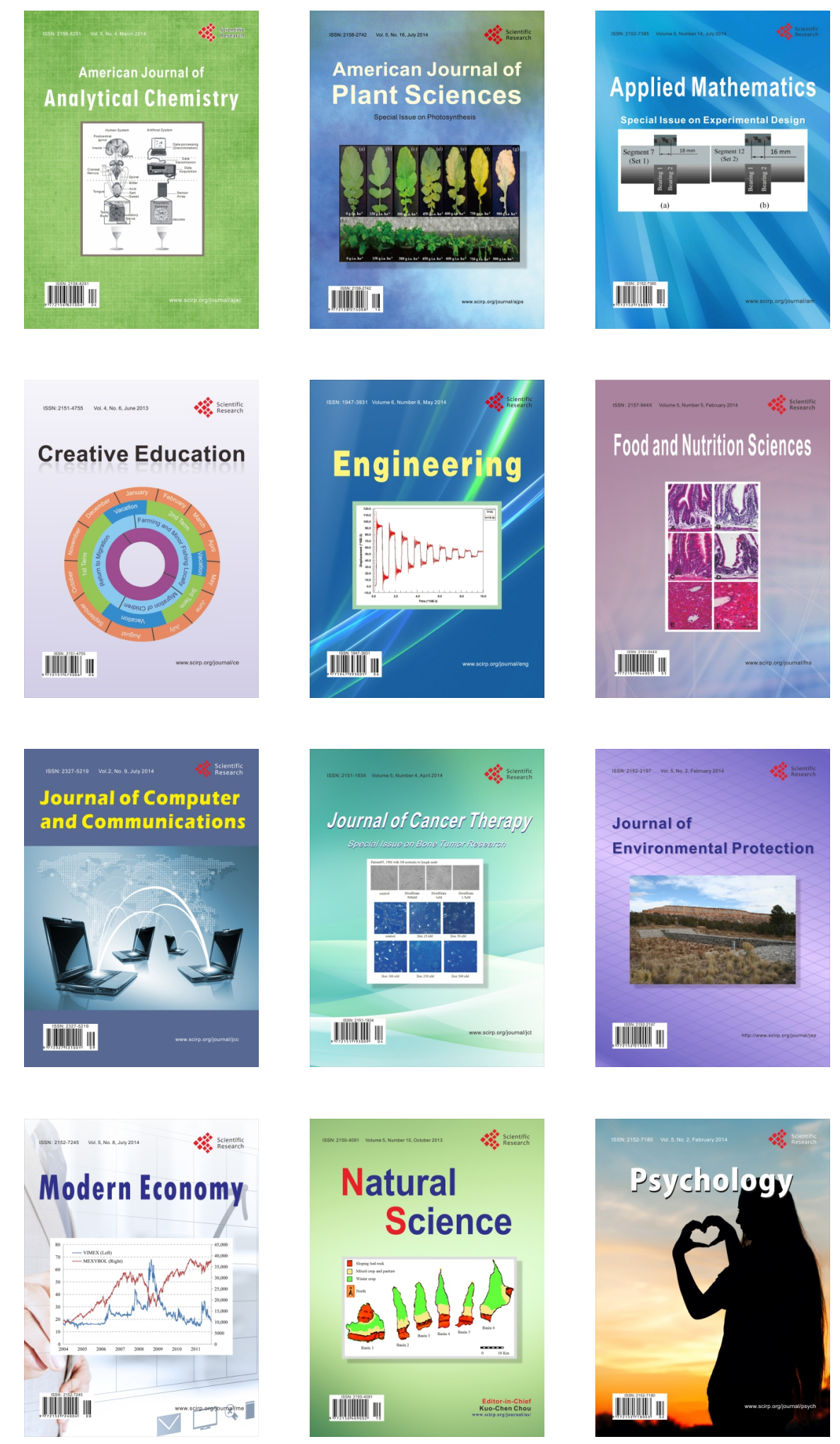